1. Bartsch C, Kuhn J. Tiefe Hirnstimulation bei Sucht, Anorexie und Zwang. Rationale, klinische Ergebnisse und ethische Implikationen. Nervenarzt 2014; 85 (2): 162-8

2. Kohl S et al. Deep brain stimulation for treatment-refractory obsessive compulsive disorder: a systematic review. BMC Psychiatry 2014; 14: 214
3. Luyten $L$ et al. Electrical stimulation in the bed nucleus of the stria terminalis alleviates severe obsessive-compulsive disorder. Mol Psychiatry 2015, Aug 25 [epub ahead of print]

4. Kohl S et al. The bed nucleus: a future hot spot in obsessive compulsive disorder research? Mol Psychiatry 2016, Apr 12. [epub ahead of print]

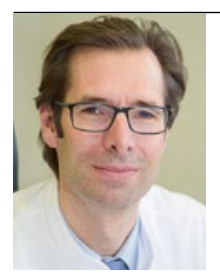

Prof. Dr. med. Jens Kuhn Klinik für Psychiatrie, Johanniter Krankenhaus, Oberhausen \& Klinik für Psychiatrie, Uniklinik Köln

\title{
Ist die rTMS eine Therapieoption bei der Zwangserkrankung?
}

\section{Die repetitive transkranielle Magnetstimulation findet vermehrt Einsatz bei therapieresistenten Zwangserkrankungen. Die supplementär motorische Area scheint ein vielversprechendes Zielgebiet dafür zu sein.}

$\mathrm{V}$ on den Patienten mit Zwangserkrankungen zeigen $40-60 \%$ ein nur unzureichendes Ansprechen auf eine Standardtherapie. Bei therapieresistenten Zwangserkrankungen kommt vermehrt die repetitive transkranielle Magnetstimulation (rTMS) zum Einsatz. Trotz häufiger Anwendung ist die Frage nach den optimalen Stimulationsparametern und dem Stimulationsort nach wie vor offen. Als Zielregionen sind der dorsolaterale präfrontale und der orbitofrontale Kortex sowie die supplementär motorische Area (SMA) in der Diskussion. In der aktuellen Arbeit von Pallanti et al. [1] wird die SMA stimuliert, die sich im mittleren Teil der Brodmann Area 6 im Bereich des oberen hinteren Frontallappens befindet und eine wichtige Rolle in der Planung komplexer motorischer Handlungsabläufe spielt. Sie wird auch bei der Vorstellung und dem geistigen Nachvollziehen von Handlungsabläufen aktiv. Defizite in der Response-Kontrolle und Inhibition spielen eine wichtige Rolle in der Pathophysiologie der Zwangserkrankung. Die erhöhte Aktivierung der SMA bei Zwangserkrankten und deren Angehöriger bei der Bearbeitung einer Stop-Signal-Aufgabe sind möglicherweise Ausdruck mangelnder Inhibition [2].

Bei den Probanden handelte es sich um 25 Patienten mit therapierefraktärer Zwangserkrankung. Als therapieresistent galten Patienten mit mindestens zwei Therapieversuchen über acht Wochen mit zwei verschiedenen SSRI, Therapieversuch mit Clomipramin und ein Versuch mit Verhaltenstherapie. Die rTMS-Behandlung erfolgte über drei Wochen. Als Vergleichsgruppe dienten 25 Patienten, die ebenfalls therapieresistent auf ein SSRI waren. Die Therapie wurde mittels Antipsychotikum augmentiert. Die Studienrater waren verblindet, das Studiendesign war open label. Die rTMS erfolgte bei $1 \mathrm{~Hz} / 1.200$ Pulsen täglich mit 100\% Motorschwelle. Beide Gruppen waren bezüglich Geschlecht und Schwere der Erkrankung gut vergleichbar. Die rTMSGruppe war aber signifikant älter. Als Messinstrumente wurden etablierte psychometrische Skalen (Y-BOCS, HAM-D, HAM-A) eingesetzt. Anzumerken ist, dass die Medikation über mindestens acht Wochen unverändert sein musste. Die Medikation war aber nicht einheitlich und auch in der rTMS-Gruppe gab es Patienten mit Antipsychotika.

Die rTMS zeigte sich der Augmentation durch Antipsychotika überlegen, so sprachen zwei Drittel der Patienten auf die rTMS-Behandlung an, dagegen nur ein Viertel auf die Augmentation durch Antipsychotika. Sieben Patienten der rTMS-Gruppe respondierten, davon remittierten drei. Hingegen sprachen nur sechs Patienten der Antipsychotikagruppe an, keiner remittierte.

\section{Kommentar}

Die Studie bietet einen interessanten Beitrag zur Behandlung der therapieresistenten Zwangserkrankung durch die rTMS über der SMA. Auf den ersten Blick ist das Ergebnis recht eindrucksvoll. Es ist jedoch zu be- denken, dass die Ergebnisse vorläufig sind und ein Vergleich mittels einer sham-rTMSBehandlung erfolgen muss. So lässt sich auch vermuten, dass die tägliche Behandlung durch rTMS einen höheren Placeboeffekt aufweist, als die Gabe eines Antipsychotikums. Diesen Aspekt diskutieren auch die Autoren der Arbeit. Als weiterer Kritikpunkt muss erwähnt werden, dass die Patienten keine einheitliche Medikation hatten, auch wenn diese über mindestens acht Wochen vor Studieneintritt unverändert war. Für eine Therapieempfehlung über den Einsatz der rTMS bei der Zwangserkrankung sind weitere prospektive, kontrollierte Studien in einer ausreichenden Patientenzahl notwendig. Die gegenwärtigen Studien sind noch zu heterogen bezüglich der Stimulationsparameter und der Patientengruppen, um eine Empfehlung abgeben zu können. Generell kann aber festgehalten werden, dass die SMA ein vielversprechendes Zielgebiet für Stimulationsmethoden bei Zwangserkrankungen ist. Es scheint, hier liegen bessere Ergebnisse vor als bei der Stimulation anderer Zielregionen [3].

1. Pallanti $S$ et al. Better than treated as usual: Transcranial magnetic stimulation augmentation in selctive serotonin reuptake inhibitor-refactory obsessive-compulsive disorder mini review an pilot open label trial. J Psychopharmacology 2016, Feb 3 [epub ahead of print]

2. Yücle $M$ et al. Functional and biochemical alterations of the medial frontal cortex in obsessive-compulsive disorder. Arch Gen Psychiatry 2007; 64 (8): 946-55

3. Saba $\mathrm{G}$ et al. Transcranial cortical stimulation in the treatment of obsessive-compulsive disorders: efficacy studies. Curr Psychiatry Rep 2015; 17 (5): 36

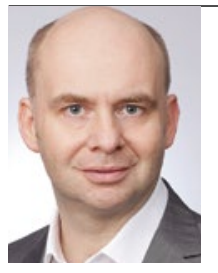

Dr. med. Jan Di Pauli Erwachsenenpsychiatrie, Landeskrankenhaus Rankweil, Österreich 